\title{
Design, construction and operation features of high-rise structures
}

\author{
Alexey Mylnik ${ }^{1,{ }^{*}}$ Vladimir Mylnik ${ }^{1}$, Elena Zubeeva ${ }^{1}$, Olga Mukhamedzhanova ${ }^{2}$ \\ ${ }^{1}$ Moscow Aviation Institute (National Research University), Volokolamskoe highway, 4, Moscow, \\ 125993, Russia; \\ ${ }^{3}$ Moscow State University of Civil Engineering, Yaroslavskoe sh. 26, Moscow, 129337, Russia
}

\begin{abstract}
The article considers design, construction and operation features of high-rise facilities. The analysis of various situations, that come from improper designing, construction and operation of unique facilities, is carried out. The integrated approach is suggested, when the problems of choosing acceptable constructional solutions related to the functional purpose, architectural solutions, methods of manufacturing and installation, operating conditions for unique buildings and structures are being tackled. A number of main causes for the emergency destruction of objects under construction and operation is considered. A number of measures are proposed on the basis of factor classification in order to efficiently prevent the situations, when various negative options of design loads and emergency impacts occur.
\end{abstract}

\section{Introduction}

At the present moment, Russian government is developing special trends in the field of urban policy. The implementation of projects for the construction of unique buildings and structures is an urgent issue, due to the modernization and improvement of construction technologies, design, construction and operation methods of real estate, the use of modern equipment, products and materials, as well as the need to improve and enrich the existing architectural style.

In accordance with the resolution of the Government of Moscow dated 28.12.2005, a group of high-rise buildings, with a height of more than $75 \mathrm{~m}$, combined with a general functional-planning and architectural-spatial solution, including a mandatory list of local service facilities is defined as a multifunctional high-rise building.

\section{Materials and Methods}

In terms of the great responsibility for the design of high-rise buildings, the following features should be considered:

1. Effective assessment of engineering and geological conditions.

\footnotetext{
* Corresponding author: vint215@yandex.ru
} 
When choosing a site for the construction of a high-rise building, it is necessary to make a thorough assessment of the engineering and geological conditions, as well as the possibility of safe construction of the building, taking into account the possible effects of dangerous geological and engineering-geological processes (karst-suffosion, landslide, etc.).

2. Survey of the foundations of buildings, that are located in the zone of potential construction.

When constructing a high-rise building in a built-up area, it is necessary to perform surveys of the foundations of buildings and structures that fall within the zone of influence of high-rise construction, as well as carry out a forecast of changes in the stress-strain behavior of the soil mass and the ground water dynamics [1].

3. Consideration of design features of high-rise buildings.

3.1. Providing spatial rigidity of the structural system.

The spatial rigidity of high-rise buildings is ensured by:

- developed in plan and symmetrically located diaphragms and structural cores;

- box-shaped structural systems with load-bearing external walls along the whole perimeter of the building;

- Rigid discs' slab, combining vertical load-bearing structures and performing the functions of horizontal stiffening diaphragms under the effect of wind or seismic loads;

- rigid nodal interfaces between load-bearing structures;

- horizontal stiffener rings in the level of technical floors, providing joint work on the bend of all vertical load-bearing structures of the building.

3.2. Providing protection against the progressive collapse of load-bearing structures.

High-rise buildings should be protected from progressive collapse in case of local destruction of load-bearing structures as a result of natural and man-caused emergencies.

Natural emergencies include dangerous meteorological phenomena, the formation of karst craters and dips in the foundations of buildings [2].

Man-caused emergencies include explosions outside or inside the building, fires, accidents or significant damage to load-bearing structures due to defects in materials, poorquality work, etc.

3.3. Additional calculations of various kinds of loads applied on the structures of highrise buildings.

In high-rise buildings, additional short-term loads are taken into account such as:

- the load from the rescue capsule of the fire-fighting helicopter to cover;

- Load from vehicles, including fire trucks;

- load on the coverings of the stylobation (the upper part of the basement) and the underground parts of the buildings that protrude beyond the dimensions of the main volume.

In addition, a special assessment of wind and aerodynamic loads is given as part of design documentation.

It is necessary to take into account the various extra-impacts of the wind, including:

- average and pulsation components of the design wind load;

- the maximum values of the wind load acting on the enclosing structures;

- resonance vortex excitation;

- effects that excite aerodynamic unstable oscillations;

- effects that cause violations of comfort conditions of pedestrian areas, which are adjacent to the building.

Values of these effects, aerodynamic coefficients of forces, moments, internal and external pressures, evaluation of resonance vortex excitation, usually should be taken on the basis of testing models (mockups) of high-rise buildings (including existing buildings) in specialized wind tunnels [3]. 
It is necessary to ensure the reduction of wind flows arising at the first floors of the high-rise building and the adjacent facilities. Limiting horizontal displacements of the top of high-rise buildings and accelerating the fluctuations in overlaps of the upper floors are taken into account when the pulsation component of the wind load acts in order to ensure a comfortable stay of people in high-rise buildings.

3.4. Special technical solutions for facade structures and openings.

It is necessary to resolve issues related to the construction of hinged facades, their installation, repair and operation as well as structure and operation of non-opening window fillings of the upper floors.

Structures of windows and hinged translucent facade structures and their attachment to load-bearing structures should be calculated by strength and deformability to the effect of wind loads.

4. Calculation of seismic effect on buildings.

The calculation is carried out on the basis of the corresponding maps of seismic microzoning.

5. Thermo-technical calculations of outer walls.

Calculations of heat losses by external enclosing structures, air balance of high-rise buildings, outdoor air parameters in the places of air intake devices, etc. are performed with account of changes in the speed and temperature of the outdoor air throughout the entire height of the buildings. The parameters of outdoor air are taken under consideration of the following factors:

- decrease of the air temperature at the altitude of more than $150 \mathrm{~m}$;

- increase of wind speed during the cold season;

- appearance of powerful convective heat flux affecting the facades of the building, that is irradiated by the sun.

6. Performance of additional firefighting measures.

The high-rise building has a special degree of fire resistance. It is divided into fire compartments in height. According to some experts, the division into vertical compartments is a matter of argument, since the flames on the ascending air streams bypass not only fire barrier slabs, but also technical floors, and the construction of firefighting visors at high altitudes is also a dubious solution, since the visor will be small due to snow load and rising air currents. High-rise buildings should be equipped with an automatic fire alarm system. Automatic fire detectors (or stand-alone fire detectors having an outlet to the fire alarm system) should be installed in all rooms except for premises with wet processes [4].

7. Performance of additional measures for the engineering systems and communication lines.

Engineering systems of high-rise buildings have a number of features:

- availability of at least two network inputs;

- additional technical premises;

- special systems to ensure safety of the building and premises to set them;

- special requirements for thermal protection of floors, which are located above $75 \mathrm{~m}$;

- heat supply from two independent sources, and in some cases - a special boiler room;

- division of heating systems into zones to avoid excess pressure in low zones;

- additional fire pumping and spare tanks on technical floors are required for fire water supply;

- electricity is provided from two independent sources, in the presence of a third emergency source, which is a diesel power plant.

8. Performance of additional measures for the installation of garbage chutes and elevator shafts. 
For high-rise buildings, there have to be special requirements for the arrangement of garbage chutes and elevator shafts. Requirements for permissible deviations of different shafts in height are stricter than for massive housing buildings.

9. Implementation of an automated building management system.

Automated building management system is required for high-rise buildings, which performs centralized monitoring, dispatching and management of engineering systems. The main functions of automated complexes, communication and information systems, which high-rise buildings are equipped with, are safety, life support of the building and provision of technological functionality of functional blocks as well as comfort of people. Technical systems must be combined into complexes that ensure the exchange of information between systems in order to eliminate redundancy and ensure the coherence of work. A flexible, freely programmable distribution system with an automated workplace, where only highly trained specialists can work, should be organized [5].

10. The arrangement of additional rooms for the placement of technological equipment for the following purposes:

- technological equipment of the departments of city emergency services

- a stationary monitoring station for load-bearing structures of a building;

- equipment for the central control point of the building security system.

\section{Results}

The following precedents for design, construction and operation of unique facilities can be distinguished dwelling on their specific features and development trends in this field:

1. Emergency collapse of building structures during construction and operation;

2. Damage (theft) of expensive property (products, materials, equipment) at the construction site;

3. Inconsistency of the initial permissive documentation to the legislation and norms in the field of construction of unique real estate objects;

4. Inconsistency of the construction of a unique facility to the legislation in the field of environmental safety.

Unique facilities belong to the construction objects of a high level of responsibility. Therefore, it is necessary to carry out additional measures at all stages from design to the facility abandonment in order to ensure the safe operation of the building.

A number of main causes for the emergency destruction of the unique facilities during construction and operation can be highlighted:

1. Preventive measures to avoid accidents, ensure the safety of constructed and operated facilities, taken by the executive and supervisory authorities, as well as construction and operation organizations, enterprises and associations, are insufficient. The number of accidents does not decrease, when their severity grows and the amount of victims increases;

2. The main share of accidents falls on the buildings and facilities in operation and accounts for $85 \%$ of the total number of registered accidents. The materials of the investigation show that the main causes of accidents at the operated facilities are gross violations of the rules for the technical operation of buildings and structures;

3. There is no proper control of the technical condition of buildings and structures.

The issue of more efficient control of the technical condition of a unique building or structure is one of the main issues in the system of integrated security of functioning of construction projects. Especially, it is urgent in terms of the large number of accidents and collapses that have occurred in recent years:

- the dome of the Transvaal Park in Moscow, 2004 (Fig. 1.1);

- section of the airport in France, Paris, 2005 (Fig. 1.2);

- roofing of ice rinks in Germany and Austria, 2006 (Fig. 1.3); 


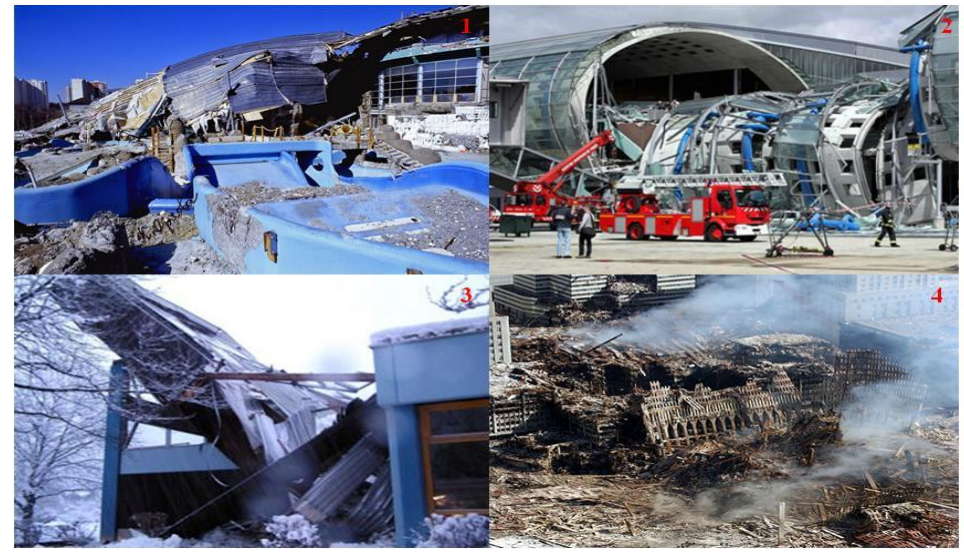

Fig. 1. Consequences of accidents.

The consequence of such accidents are not only economic losses, but in many cases there are dire environmental and social consequences. A detailed analysis of the causes and consequences contributes to reducing the number of accidents.

Analysis of the circumstances of emergency destruction of structures of unique buildings allows distinguishing the following main causes:

- violation of the operation rules;

- defects at the construction stage and deviations from the design;

- violations of the technology of performance of works during construction, reconstruction and repairs;

- low quality of manufacturing of building structures.

Moreover, the following factors should be noted:

1. The development of the project and design features of unique structures, as a rule, is based on the experience and engineering foreknowledge of the designer, and the application of the acquired skills does not always give an accurate idea of the design model.

2. Imperfections, defects and damages of load-bearing elements of a structure that occur during manufacture, transportation and installation, a seemingly insignificant disparity between the final geometry of the structure and the idealized design configuration, which for traditional structures would not be significant, but for structures with unique parameters result in the occurrence of serious additional efforts and displacements.

3. The change in the mechanical characteristics of the used materials (aging, relaxation, creep, etc.), operational factors, geological conditions, that take place over time, become a serious matter due to the significant regulatory lifetime of such structures.

Thus, modern approaches to the design of unique buildings and structures provide only the initial constructional safety of the facility, i.e. design structural safety at the time of commissioning of the facility.

The constructional safety of this facility type during operation can significantly change since the design stage for the listed reasons. Firstly, due to possible deviations from design decisions during the construction of the facility. Secondly, due to operational factors, where there are changes in the object itself, in particular, as a result of material aging, corrosion of metal structures, space planning changes associated with alterations, extensions, reconstructions, etc. Thirdly, due to changes in external factors, changes in the level of surface water; physical and mechanical parameters of the foundation soil, the occurrence of dynamic impacts, the movement of soil in connection with the nearby new construction of aboveground and underground structures, etc.

Fourth, due to the "human" factor: mistakes, ignorance, negligence of the staff. 
It should be borne in mind that accidents at unique buildings and structures, that are caused by their collapse, can occur according to two patterns:

- either with a gradual accumulation of stresses and deformations and subsequent collapse of load-bearing structures;

- or fleetingly (progressing, avalanche-like collapse) with possibly even a short-term but significant overload of an important supporting structural element, from the destruction of which, subsequent progressive collapse can happen. An example of this was the collapse of the World Trade Center buildings in New York City in 2001 (Fig. 1.4), where such a situation was not foreseen at the design stage.

In order to effectively prevent the first collapse pattern, it is necessary to have a regulatory framework for monitoring unique structures, that are under development.

- At the present time, protection in the second collapse pattern time can only be reliable calculation of load-bearing structural elements and appropriate structural measures to ensure the inadmissibility of a progressive collapse, since neither of the existing systems of deformation control of building structures can help at such collapse. If the process has started, even preliminary detection does not allow any action to be taken to prevent or rescue people and equipment because of its explosion-like fleetness.

\section{Discussions}

Diagnostics of load-bearing structures of buildings at an early stage becomes especially important for unique, technically complex high-rise buildings, since it is not easy to control the change in the stress-strain state of load-bearing structures through traditional methods of visual and instrumental inspection. In addition, in high-rise construction, a significant influence on the stress-strain state of the bearing structure is exerted by inclines and wind effects, which creates a greater dispersion of the places of accumulation of deformation stresses [6]. To date, there is no generally accepted scientifically grounded approach or the practice of designing unique structures that maintain structural integrity for various options of design loads and emergency effects.

\section{Conclusion}

In the context of large-scale implementation of projects for the construction of unique facilities, as well as drawbacks and a number of problems related to the regulation of the design, construction and operation of these facilities, judicial situations arise that require the expert examination of the accident which occurred with the object.

\section{References}

1. R.S. Golov, V.V. Shilov, S.A. Silantiev, ASEE International Forum (2017)

2. A. Jones, G. Fallon, R. Golov, European Business Review, 12, 187-197 (2017) doi. 10.1108/09555340010336871

3. E. Kiseleva, M. Nekrasova, M. Mayorova, M. Rudenko, V. Kankhva, International Review of Management and Marketing, 6, 95 (2016)

4. I. Lukmanova, M. Mishlanova, International Journal of Economics and Financial Issues, 5, 208-216 (2012)

5. A. Konikov, G. Konikov, Procedia Engineering, 165, 1052-1056 (2016)

6. V. Gasilov, N. Anisimova, I. Provotorov, MATEC Web of Conferences, 106, 08035 (2017) DOI: 10.1051/ mateconf / 201710608035. 